\title{
PHOTOABSORPTION OF GAMMA RAYS IN RELATIVISTIC JETS
}

\author{
CHARLES D. DERMER \\ Code 7653, Naval Research Laboratory \\ 4555 Overlook Ave. SW, Washington, DC 20375-5352 USA \\ E-mail: dermer@gamma.nrl.navy.mil
}

\begin{abstract}
A derivation of the $\gamma \gamma \rightarrow \mathrm{e}^{+} \mathrm{e}^{-}$optical depth for $\gamma$ rays produced in a comoving spherical emitting region is presented. Employing a simplified expression for the $\gamma \gamma$ absorption cross section, analytic expressions for the minimum Doppler factor implied by the requirement of $\gamma$-ray transparency are derived for a broken powerlaw spectrum of target photons which are isotropically distributed in the comoving frame. Application to specific systems is illustrated.
\end{abstract}

\section{Introduction}

One particularly powerful probe of relativistic motions of AGN and GRB jets, as revealed by the EGRET instrument on the Compton Observatory, is the use of $\gamma$-ray observations to infer minimum Doppler factors of the radiating plasma. The basic idea is simple: the measured FWHM variability time scale $t_{v a r}$ of a blazar flare or GRB pulse implies a maximum comoving radius of the emitting region from causality considerations. The measured flux and redshift implies the corresponding density of photons which provide targets for $\gamma \gamma \rightarrow \mathrm{e}^{+} \mathrm{e}^{-}$pair production attenuation of $\gamma$ rays. The requirement that the emitting region have small optical depth for observed $\gamma$ rays places a lower limit on the Doppler factor $\delta$ of the emitting region. In many cases, such arguments indicate that the radiating plasma in blazar and GRB jets must be relativistic 12 .

The dramatic improvements in sensitivity of the upcoming GLAST mission and the ground-based imaging air Cherenkov telescopes VERITAS and HESS over previous instruments offer the opportunity to place better limits on $\delta$, to monitor changes of $\delta$ in a given source, and to compare $\delta$ between members of different source classes, e.g., BL Lac objects and flat spectrum radio quasars (FSRQs), and X-ray rich, short duration, and classical GRBs. Because GLAST is a scanning mission, blazar flares can be correlated with radio outflows to infer the locations of the sites of $\gamma$-ray emission. The $\gamma$-ray observations, coupled with correlated multifrequency data, will provide knowledge of the jet-disk connection, jet dynamics, and radiation fields in the vicinity of the jet. For these reasons, it seems appropriate to revisit the problem of $\gamma$-ray photoabsorption in relativistic jets.

\section{Derivation of the Minimum Doppler Factor}

We consider a uniform spherical radiating blob of volume $V_{b}^{\prime}=4 \pi r_{b}^{\prime 3} / 3$, where primes refer to the comoving frame. The measured $\nu F_{\nu}$ flux is related to the 
comoving emissivity according to the relation

$$
\nu F_{\nu}=f_{\epsilon} \cong \frac{\delta^{4} V_{b}^{\prime}}{d_{L}^{2}} \epsilon^{\prime} j^{\prime}\left(\epsilon^{\prime} ; \vec{\Omega}^{\prime}\right) \rightarrow \frac{\delta^{4} V_{b}^{\prime}}{4 \pi d_{L}^{2}} \epsilon^{\prime} j^{\prime}\left(\epsilon^{\prime}\right)
$$

where $j^{\prime}\left(\epsilon^{\prime}, \vec{\Omega}^{\prime}\right)$ is the comoving emissivity, $d_{L}=10^{28} d_{28} \mathrm{~cm}$ is the luminosity distance, $\epsilon=h \nu / m_{e} c^{2}=\delta \epsilon^{\prime} /(1+z)$ is the dimensionless photon energy, $z$ is the source redshift, $\vec{\Omega}$ is the direction vector, and the final expression in eq. (11) assumes isotropic emission in the comoving frame. Eq. (1) is an approximation because we have implicity assumed that the light travel time across the blob is smaller than the duration of the flare in order to avoid integrations over different portions of the emitting plasma, consistent with causality arguments for a discrete intense flare or pulse. The expression $d t=(1+z) d t^{\prime} / \delta$ relating observer and comoving differential times implies $r_{b}^{\prime} \lesssim c t_{v a r}^{\prime}=c \delta t_{\text {var }} /(1+z)$.

The comoving spectral energy density $u_{\epsilon^{\prime}}^{\prime}=m_{e} c^{2} \epsilon^{\prime 2} n^{\prime}\left(\epsilon^{\prime}\right)$ is related to the isotropic emissivity $j^{\prime}\left(\epsilon^{\prime}\right)$ through the relation $\epsilon^{\prime} j^{\prime}\left(\epsilon^{\prime}\right) \cong u_{\epsilon^{\prime}}^{\prime} /\left\langle t_{\text {esc }}^{\prime}\right\rangle \cong c u_{\epsilon^{\prime}}^{\prime} / r_{b}^{\prime}$, where $\left\langle t_{\text {esc }}^{\prime}\right\rangle$ is the mean photon escape time and $n\left(\epsilon^{\prime}\right)$ is the spectral photon number density. Thus

$$
u_{\epsilon^{\prime}}^{\prime} \cong \frac{3 d_{L}^{2}}{c r_{b}^{\prime 2} \delta^{4}} f_{\epsilon}=\frac{3 d_{L}^{2}}{c r_{b}^{\prime 2} \delta^{4}} f_{\epsilon_{p k}}\left[x^{a} H(1-x)+x^{b} H(x-1)\right],
$$

where $x=\epsilon / \epsilon_{p k}=\epsilon^{\prime} / \epsilon_{p k}^{\prime}, H(u)$ is a Heaviside function, and we have approximated the $\nu F_{\nu}$ spectrum by a broken power law with peak energy flux $f_{\epsilon_{p k}}$ at $\epsilon=\epsilon_{p k}$ with $\nu F_{\nu}$ indices $a(>0)$ and $b(<0)$ at low and high frequencies, respectively.

The photoabsorption optical depth for a $\gamma$-ray photon with energy $\epsilon_{1}$ in a radiation field with spectral photon density $n\left(\epsilon^{\prime}, \mu^{\prime} ; r^{\prime}\right)\left(\approx n\left(\epsilon^{\prime}\right) / 2\right.$ for a uniform isotropic radiation field in the comoving frame) is 3

$$
\begin{gathered}
\tau_{\gamma \gamma}\left(\epsilon_{1}^{\prime}\right)=\int_{r_{1}^{\prime}}^{r_{2}^{\prime}} d r^{\prime} \int_{-1}^{1} d \mu^{\prime}\left(1-\mu^{\prime}\right) \int_{2 / \epsilon_{1}^{\prime}\left(1-\mu^{\prime}\right)}^{\infty} d \epsilon \sigma_{\gamma \gamma}\left[\epsilon^{\prime} \epsilon_{1}^{\prime}\left(1-\mu^{\prime}\right)\right] n\left(\epsilon^{\prime}, \mu^{\prime} ; r^{\prime}\right) \\
\cong r_{b}^{\prime} \int_{0}^{\infty} d \epsilon^{\prime} \sigma_{\gamma \gamma}\left(\epsilon^{\prime}, \epsilon_{1}^{\prime}\right) n\left(\epsilon^{\prime}\right) .
\end{gathered}
$$

Using the approximation $\frac{4}{\gamma \gamma} \sigma_{\gamma}\left(\epsilon_{1}^{\prime}\right) \cong \frac{1}{3} \sigma_{\mathrm{T}} \epsilon^{\prime} \delta\left(\epsilon^{\prime}-2 / \epsilon_{1}^{\prime}\right)$ for the cross section and making the substitutions in eq. (3) gives

$$
\tau_{\gamma \gamma}\left(\epsilon_{1}\right)=\tau_{\gamma \gamma}^{p k}\left[\left(\frac{\epsilon_{1}}{\epsilon_{1}^{p k}}\right)^{1-b} H\left(\epsilon_{1}^{p k}-\epsilon_{1}\right)+\left(\frac{\epsilon_{1}}{\epsilon_{1}^{p k}}\right)^{1-a} H\left(\epsilon_{1}-\epsilon_{1}^{p k}\right)\right],
$$

where

$$
\tau_{\gamma \gamma}^{p k} \cong \frac{\sigma_{\mathrm{T}} d_{L}^{2} f_{\epsilon_{p k}}}{4 m_{e} c^{4} t_{v a r} \delta^{4} \epsilon_{p k}} \text { and } \epsilon_{1}^{p k}=\frac{2 \delta^{2}}{(1+z)^{2} \epsilon_{p k}} .
$$

Requiring that $\tau_{\gamma \gamma}\left(\epsilon_{1}\right)<1$ so that the emission region is transparent to $\gamma$ rays, we obtain a minimum Doppler factor given by

$$
\delta>\left[\frac{\sigma_{\mathrm{T}} d_{L}^{2} f_{\epsilon_{p k}}}{4 m_{e} c^{4} t_{v a r} \epsilon_{p k}^{A}}\left(\frac{(1+z)^{2} \epsilon_{1}}{2}\right)^{1-A}\right]^{1 /(6-2 A)} .
$$


In eq. (6), $A=b$ if $\epsilon_{1}<\epsilon_{1}^{p k}$, and $A=a$ if $\epsilon_{1}>\epsilon_{1}^{p k}$ (note that $A=1-\alpha$ where the energy index $\alpha$ is defined by the relation $\left.F_{\nu} \propto \nu^{-\alpha}\right)$.

\section{Application to Observations}

We first apply eqs. (5) and (6) to FSRQs such as 3C 279, PKS 0528+134, or CTA 102. OSSE and COMPTEL observations 5 of bright blazars show that $f_{\epsilon_{p k}}=$ $10^{-10} f_{-10}$ ergs $\mathrm{cm}^{-2} \mathrm{~s}^{-1}$ with $f_{-10} \gtrsim 1$, and $f_{-10} \approx 10$ during bright blazar flares. The $\nu F_{\nu}$ flux peaks at $\epsilon_{p k} \sim 1-100$. Photons observed at $100 \mathrm{MeV} / \mathrm{GeV}$ energies (i.e., $\epsilon_{1}=1960 E_{G e V} \gtrsim 200-2000$ ) would therefore be preferentially absorbed by photons with energies below the peak of the $\nu F_{\nu}$ spectrum, so that the case with $A=a$ applies, and $a \sim 0.2-1$. Taking $a=0.5$ for illustration gives

$$
\delta \gtrsim 6.8\left[\frac{d_{28}^{2} f_{-10}}{t_{4}}\left(\frac{1+z}{2}\right)\right]^{1 / 5}\left(\frac{E_{G e V}}{\epsilon_{p k}}\right)^{1 / 10} .
$$

Here we have used $t_{4}=t_{\text {var }}(\mathrm{s}) / 10^{4} \mathrm{~s} \sim 1$ as a fiducial because $G L A S T$ will be able to detect variability from flares at the level of $f_{-10} \sim 1$ on this timescale 6 . For bright blazar flares from distant FSRQs, GLAST could set minimum values of $\delta$ exceeding $\sim 20$, comparable to the largest values inferred from radio observations of superluminal motion.

We next consider TeV blazars $\left(\epsilon_{1}=1.96 \times 10^{6} E_{T e V}\right)$ such as Mrk 421 or Mrk 501 at a distance of $\approx 140 \mathrm{Mpc}$. TeV photons from sources with $\delta \approx 10$ preferentially pair produce with $\sim 1 \mathrm{keV}$ synchrotron photons, the X-ray synchrotron spectrum can exceed $f_{-10} \approx 1$, and the flare timescale $t_{4}$ may be as low as 0.1 , or even lower, depending on results from the next generation of ground-based $\gamma$-ray telescopes. Using $A=a=b=0$ for simplicity (a flat $\nu F_{\nu}$ synchrotron spectrum) gives

$$
\delta \gtrsim 10.9\left[\left(\frac{d_{L}}{140 \mathrm{Mpc}}\right)^{2} \frac{f_{-10} E_{T e V}}{t_{4}}\right]^{1 / 6} .
$$

Shorter flares and brighter synchrotron fluxes will potentially imply values of $\delta \gtrsim 15$.

Our results can also be applied to GRBs. The main difference here is that the geometry of emission for GRBs is generally considered to be a spherical blast wave that is uniform within the Doppler beaming cone. This geometry for GRB emission lacks strong observational support, so a blob geometry might equally well apply to a GRB; in any case, the differences between blast-wave and blob geometries do not make a great deal of difference in the resultant expressions for the pair-production opacities (Dermer 2004, in preparation).

The prompt hard X-ray/soft $\gamma$-ray emission of a GRB, which we assume here to be nonthermal synchrotron radiation, has $\epsilon_{p k} \sim 1$. GRBs with peak flux $f_{\epsilon_{p k}}=$ $10^{-6} f_{-6}$ ergs $\mathrm{cm}^{-2} \mathrm{~s}^{-1}$, with $f_{-6} \gtrsim 1$, occur every $2-4$ weeks over the full sky. Anticipating that $\delta_{100}=\delta / 100 \approx 1$ for GRBs, we see that $\epsilon_{1}^{p k} \approx 5000 \delta_{100}^{2} /\{[(1+$ $z) / 2]^{2} \epsilon_{p k}$, so that observations of $\mathrm{GeV}$ photons from GRBs generally favor the case $\epsilon_{1} \lesssim \epsilon_{1}^{p k}$. In other words, GeV photons from GRBs are preferentially attenuated by photons on the high-energy portion of the synchrotron spectrum. For illustration, we let $A=b=-1 / 2$ for a typical value of the spectral index at $\gtrsim \mathrm{MeV}$ energies,

main: submitted to World Scientific on February 14, 2018 
and assume that the synchrotron spectrum extends to $\mathrm{GeV}$ energies, giving

$$
\delta \gtrsim 177\left[\frac{f_{-6} d_{28}^{2}}{t_{\text {var }}(\mathrm{s})}\left(\frac{1+z}{2}\right)^{3}\right]^{1 / 7}\left[\epsilon_{p k} E_{G e V}^{3}\right]^{1 / 14}
$$

Thus we can expect GLAST observations of GRBs to infer values of $\delta \gg 200$ in the brightest and most variable GRBs, and potentially infer differences in values of $\delta$ between "classical" GRBs and X-ray flashes, which are predicted to be low $\delta$-factor GRBs?

\section{Discussion}

The results here are in essential agreement with previous treatments, taking into account the different approximations made for the cross sections $\frac{12}{2}$. Note an implicit co-spatial assumption in the derivation, namely that the $\gamma$-rays are formed in the same region as the lower energy target photons. Without this assumption, only much smaller values of minimum Doppler factors can be confidently asserted, which depend more on observations at $\mathrm{MeV}$ energies rather than at $\mathrm{GeV}$ or $\mathrm{TeV}$ energies 8 . To demonstrate the reliability of the co-spatial assumption, and to measure the spectrum of photons that attenuate the $\gamma$ rays, GLAST and ground-based $\gamma$-ray observations of blazars and GRBs should be correlated with observations made by X-ray/soft $\gamma$-ray detectors such as INTEGRAL, Chandra, XMM Newton and Swift.

\section{Acknowledgments}

I thank Drs. Roberto Turolla and Silvia Zane for the opportunity to speak in the session on Radiative Transfer in Relativistic Astrophysics at the 10th Marcel Grossman Meeting. This work is supported by the Office of Naval Research and GLAST Science Investigation Grant No. DPR-S-1563-Y.

\section{References}

1. L. Dondi and G. Ghisellini, MNRAS 273, 583 (1995).

2. Y. Lithwick and R. Sari, Astrophys. J. 540, 555 (2001).

3. R. J. Gould and G. P. Schréder, Phys. Rev. 155, 1404 (1967).

4. A. A. Zdziarski and A. P. Lightman, Astrophys. J. 294, L79 (1985).

5. K. McNaron-Brown et al., Astrophys. J. 451, 575 (1995).

6. C. D. Dermer and B. L. Dingus, astro-ph/0312590 (2003).

7. C. D. Dermer, J. Chiang, and M. Böttcher, Astrophys. J. 513, 656 (1999).

8. C. D. Dermer and N. Gehrels, Astrophys. J. 447, 103 (1995).

main: submitted to World Scientific on February 14, 2018 